\title{
Young Adult Health Promotion: Supporting Research Design with Eye-Tracking Methodologies
}

\author{
Soussan Djamasbi and E. Vance Wilson \\ User Experience \& Decision Making Research Laboratory, \\ Worcester Polytechnic Institute, USA \\ \{djamasbi, vwilson\}@wpi . edu
}

\begin{abstract}
Despite increasing mental health problems among college undergraduate students, little work has been done to investigate factors that can improve health promotion among this population. To address this need we designed a research program that addresses health promotion toward young adults. In particular, we are interested in addressing mental health and risky health behaviors among college undergraduate students. The research reported in this study is the result of the first basic step in our research program.
\end{abstract}

Keywords: Computer-Mediated Communication (CMC), Online Persuasion, Eye-Tracking, Fixation, Involvement, Message Source, Young Adults, Health, Online Persuasion, Interpersonality Model.

\section{Introduction}

Since 2006, the percentage of college students with severe psychological problems has grown by $16 \%$. According to recent surveys of campus counseling centers, depression and anxiety are the top two mental health problems among college students [6], and these conditions can have devastating consequences. "Indeed, the second leading cause of death among college students is suicide, which accounts for about 1,100 deaths per year on campuses .... The No. 1 killer is accidents, which include accidental overdoses and drinking and driving deaths, many of which might be linked to depression and anxiety." [3].

The increasing prevalence of psychological problems among college students calls for scientific investigation of factors that can improve their health. One way to do so is by improving the effectiveness of health promotion among college students. Health promotion is defined as "the art and science of helping people discover the synergies between their core passions and optimal health, enhancing their motivation to strive for optimal health, and supporting them in changing their lifestyle to move toward a state of optimal health." [12, p. iv]. By providing support, structure, and motivation toward better health, health promotion programs aim to enable people to "increase control over, and enable, their health" $[19$, p. 1]. In the past, many health promotion 
efforts emphasized communication via mass media, including television, radio, newspapers and magazines, and targeted media, including newsletters, booklets, and videos [5]. More recently, the Internet has provided numerous new tools for health promotion, including online communication, social media, and gaming apps [9].

We recently undertook a research program that addresses health promotion toward young adults, specifically investigating mental health and risky health behaviors among college undergraduate students. As discussed above, mental health problems among this group are increasing [17] and risky health behaviors, including overweight, smoking, drinking, and risky sex, are relatively high [16]. Yet little research in the area of health promotion has been conducted among young adults due, in part, to difficulty of overcoming competing distractions of academic, social, and sporting activities, as well as the transient nature of this population's living situations [16].

Our approach in this health promotion research program is to adapt an interpersonality model of online persuasion (hereafter referenced simply as interpersonality model) to the context of young adult health promotion. The interpersonality model was developed to predict and explain message receivers' tendencies toward complying with requests they receive via email $[20,21]$. We anticipate that this model will provide theoretical direction for identifying factors that can affect young adults' intentions to visit a health-related website and to recommend such a website to their friends.

Previous research has shown that email has the capacity to be an effective tool for universities to promote student health [2]. Yet Internet sources of health-related information are known to generate relatively low levels of believability among young adults [10], and email may be inherently limited in promoting behavioral change for this reason. Our overarching goal in this research program is to identify means of enhancing effectiveness and improving consistency of email messaging in the context of health promotion to young adults.

This paper presents our initial experiences in developing a research design for an online experiment in young adult health promotion with support of an eye-tracking methodology. Research shows that eye-tracking data can provide a valuable source of information on how individuals experience and interact with information that is delivered online [4]. Thus, we argue that eye-tracking will be useful in meeting the objective to develop experimental treatments that are robust, potent, reflective of the research objectives, and free from spurious effects.

In the following sections we describe the theoretical background of the interpersonality model and the adaptations we made in developing a new research design. Additionally, we explain how we use eye tracking to test the impact of experimental treatments as well as other measures in our study.

\section{Theoretical Background}

\subsection{Interpersonality Model}

The interpersonality model presented in Figure 1 [21] proposes that message receivers evaluate characteristics of email in order to categorize whether the message is 
interpersonal, i.e., interactive communication between two or more interdependent people, or broadcast, i.e., non-interactive, one-way communication that typically is designed to address a mass audience. Email is capable of delivering interpersonal and broadcast messages, including broadcast messages that are intended to appear to be interpersonal in form. These latter messages are often referred to as spam, which message receivers are generally motivated to avoid [8].

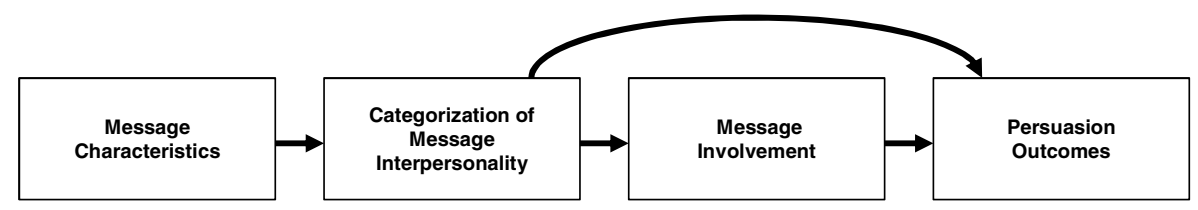

Fig. 1. Interpersonality Model of Online Persuasion

Interpersonality is determined by two factors: Message coherence, i.e., the perception that the sender's message is relevant to the receiver's situation, and personal feedback, i.e., the anticipation that the message receiver can respond to the message and receive a reply from the sender. The interpersonality model further predicts that higher message coherence and personal feedback will influence persuasion outcomes, increasing message receivers' compliance tendencies both directly and by increasing involvement with the message [21]. In the present study, we focus on two distinct persuasion outcomes: Intention by the message receiver to visit an online health resource and intention by the receiver to recommend the online health resource to a friend.

\subsection{Message Source}

Kwan et al. [10] find young adults access health-related information more from the Internet $(79 \%)$ than from parents $(66 \%)$ or health center medical staff $(56 \%)$, yet young adults rank believability of health-related information accessed via the Internet to be lower than from either of these alternative sources. Viewed from an interpersonality model perspective, this observation suggests that the source of a health promotion message may be a key message characteristic that young adults use to categorize email messages. In this study, we are interested to see whether the reaction to messages is influenced by the similarity or differences in group membership between source and receiver. In other words, is compliance more likely to happen if the message source is the organization to which the receiver belongs?

\subsection{Viewing Behavior}

While we can see many things at a glance, we can attend to only one object at a time [4]. We typically attend to objects that we can see with our focal vision [7]. The area 
covered by our focal vision, however, is relatively small. Clear and colorful vision is facilitated by only the fovea region in our eyes, which contains a densely packed array of photo photosensitive receptors [7]. To compensate for the small size of focal vision, our eyes move rapidly and continuously in the visual field from one area to another, and visual information is processed only during the short period of times that our gaze is steady [14]. These periods of fixation provide a reliable indicator of attention and cognitive processing [13].

A recent study shows that fixation also can be used to measure cognitive effort and willingness to expend cognitive effort [4]. Djamasbi et al. [4] used the duration and frequency of fixation to measure the amount of effort expended when viewing a web page. Because reading a message requires cognitive processing and willingness to expend the necessary attention to comprehend it, we propose that fixations can serve as an effective measure to capture an individual's engagement and involvement with a CMC message. In the present study, viewing behavior can help us examine whether a message from a source belonging to the same organization that the receiver is a member (in-group) is likely to produce more involvement and behavioral intentions than a message from a source that belongs to an organization other than that of the receiver (out-group).

\section{$3 \quad$ Research Method}

We used the interpersonality model $[20,21]$ as a theoretical base for our investigations, using an online survey to collect participants' responses. We operationalized Message Characteristic as whether the message came from an organization to which the receiver belongs (in-group) or an organization that is not part of the receiver's community (out-group). Categorization of Message Interpersonality was operationalized using previously validated measures of message coherence and personal feedback [20]. Persuasion Outcome was operationalized using two measures: Intention to comply with the message, using a measure previously validated by Wilson and Djamasbi [20], and intention to recommend the message to a friend, using a measure created for the present study.

To examine the effect of message source we used two treatments, as in prior studies [20, 21] participants were randomly assigned to one of the two treatments. In both treatments participants were asked to read a text message that encouraged them to visit a health-related website and complete a self-assessment for mental issues such as depression and anxiety. After completing the task (reading the message) participants were asked to complete an online survey.

We used eye tracking to test for the presence of physiological evidence distinguishing the treatments in our study as a check that treatment was successfully manipulated within the research design. In particular, we expected messages from in-group sources to receive more attention. Additionally, we wanted to explore whether the eye tracking data would help to explain effects on other constructs used in the model. We 
propose this novel use of eye tracking data can be useful in model building, as exemplified in this case by aiding in the interpretation of underlying factors that affect a young adult's behavior towards compliance with a health-related message and/or toward recommending the message to a friend. Hence, in this exploratory study we investigated the correlation between physiological measure of eye movements and the treatments used in our study as well as correlation between eye movements and survey measures that were collected and correlations among the survey measures.

\subsection{Research Treatments}

As in prior research $[20,21]$, we developed two separate research treatments which differed only in the message source information. Both treatments presented a selfassessment website hosted by a health screening organization. Treatment A was presented as an in-group message from a member of the campus Health Services staff, and Treatment B was presented as a message from the health screening organization, an "out-group" organization (see Figure 2).

\subsection{Participants}

Participants were 20 students ( 9 female and 11 male) at a major university located in the U.S. Northeast. The average age of participants was 20 years. As an incentive to attend the experiment, participants were entered in a drawing to win a $\$ 50$ gift certificate.

\subsection{Measures}

We used the survey items by Wilson and Djamasbi $[20,21]$ to measure the measures personal feedback, message coherence, message involvement and intention to comply with the message. We also designed a new measure, intention to recommend, which captures the participant's intention to recommend the website to a friend. Reliability testing showed that Cronbach's alpha measured .70 or above for all measures, indicating an acceptable level of reliability.

To capture users' reactions to the message we also tracked users' eye movements. To account for individual differences in viewing time (e.g., some people may be faster in reading than others), for each participant we calculated the participant's proportion of total time that was dedicated to viewing the body of the message.

Because our task required participants to view a CMC text message we measured fixation as steady gazes of 60 milliseconds. Studies show that people can read text with fixations as short as 50 to $60 \mathrm{~ms}$ [15]. To collect fixation data we used a Tobii X120 eye-tracker. 
Email Message From:

Subject: Please Try Out Our Self-Assessment Website

Hi everyone,

I wanted to tell you about the Self-Assessment website the Student Development \& Counseling Center has linked up with. It provides free, completely confidential guidance in response to anxiety, depression, problems with eating and alcohol use, and other mental health issues. The website is located at http://www.mentalhealthscreening.org/screening/Welcome.aspx, and it can also be found through the SDCC's website (http://www.wpi.edu/Admin/SDCC/about.html).

We recommend the Self-Assessment website highly, and we ask you to check it out. Anxiety, depression, and problems with eating and alcohol use are very common on college campuses, and getting help will make everyone's life better.

The Self-Assessment website can be a great resource for you personally, or for your friends and fellow students.

Best,

(Continues with organization name and contact information)

\section{Treatment $A$}

Email Message From: ScreeningForMentalHealth

Subject: Please Try Out Our Self-Assessment Website

Hi everyone,

We wanted to tell you about the Self-Assessment website that Screening For Mental Health has linked up with. It provides free, completely confidential guidance in response to anxiety, depression, problems with eating and alcohol use, and other mental health issues. The website is located at http://www.mentalhealthscreening.org/screening/Welcome.aspx, and it can also be found through Screening for Mental Health's website (http://mentalhealthscreening.org/).

We recommend the Self-Assessment website highly, and we ask you to check it out. Anxiety, depression, and problems with eating and alcohol use are very common on college campuses, and getting help will make everyone's life better.

The Self-Assessment website can be a great resource for you personally, or for your friends and fellow students.

Best,

(Continues with organization name and contact information)

Fig. 2. Research Treatments

\section{$4 \quad$ Results}

Contrary to our expectation, the results did not show a significant relationship between the proportion of time spent on viewing the message and whether the message 
source belonged to the receiver's community or not. Neither did the results show significant correlation between proportion of fixation duration on the message and the perception that one can receive feedback from the sender. The results, however, show significant correlation between the proportion of task time spent on viewing the message and the other survey measures. Because fixations are reliable measure of attention and cognitive processing [14,15], these correlations suggest that higher levels of cognitive processing of the message led to increased scores for message coherence, message involvement, intention to comply with the message, and intention to recommend.

Looking at the survey measures only, the results did not show significant correlation between message source and the rest of the measures. They also did not show significant correlation between personal feedback and other measures.

The results do show strong correlations between message involvement and intention to comply and intention to recommend, however. Additionally they show that intention to comply is significantly correlated with intention to recommend. These are interesting results because they are supported by the eye tracking data. That is, we found physiological evidence for the survey measures that were significantly correlated.

Table 1. Correlation Table for Eye Tracking Data and Survey Measures

\begin{tabular}{|c|c|c|c|c|c|c|}
\hline Measure & 1 & 2 & 3 & 4 & 5 & 6 \\
\hline 1. Treatment ${ }^{a}$ & - & & & & & \\
\hline $\begin{array}{l}\text { 2. Message fixation } \\
\text { ratio }\end{array}$ & -0.15 & - & & & & \\
\hline 3. Message Coherence & -0.07 & $0.57 *$ & - & & & \\
\hline 4. Personal Feedback & 0.04 & -0.05 & 0.08 & - & & \\
\hline $\begin{array}{l}\text { 5. Message } \\
\text { Involvement }\end{array}$ & -0.19 & $0.47 *$ & $0.59 * *$ & 0.44 & - & \\
\hline 6. Intention to use & -0.06 & $0.53 *$ & 0.41 & 0.26 & $0.76 * * *$ & - \\
\hline $\begin{array}{l}\text { 7. Intention to } \\
\text { recommend }\end{array}$ & -0.36 & $0.54 *$ & 0.34 & 0.19 & $0.71 * * *$ & $0.81 * * *$ \\
\hline
\end{tabular}

\section{Discussion}

Our results show that survey measures of message coherence, message involvement, intention to comply, and intention to recommend were significantly correlated with physiological measure of fixation which is an indicator of the amount of attention the message received. Additionally, the results show significant correlations among the survey measures of coherence, involvement, intention to comply, and intention to 
recommend. Taken together, these results suggest that the above measures are likely to be good candidates for investigating compliance behavior towards health-related messages.

Our results did not show significant correlation between the proportion of fixation duration on message and message source and feedback. These two measures were also not correlated with other survey measures. One possible explanation is that these measures may not be as important in compliance with health-related messages. Another possibility is that a larger sample size is needed to detect such a difference. The latter case, however, indicates that the effect sizes of these measures may be small, hence, supporting the first interpretation.

These results have important theoretical implications because they identify several measures that are likely to be helpful in investigating compliance of young adults towards health-related messages. Additionally, the results introduce the use of eye tracking for identification of relevant constructs in theoretical models. Hence the results show that eye tracking can potentially be useful in instrument development and theory building.

From a practical point of view, results involving message coherence indicate that relevance of messages is likely to have a significant impact on involvement, personal compliance, and recommendation to friends. Thus, messages developed for healthrelated issues may benefit from a market analysis and persona development which can help to increase the relevance of the message to its intended audience.

As with any experimental study, the generalizeability of the results of this study is limited by its laboratory setting and the task that it used. The controlled laboratory environment allowed us to track users' eyes, a physiological measure that provides a continuous picture of user experience. The task used in our study was designed to be appropriate to the participants of the study, namely young adults attending a university undergraduate program. Future studies are needed to test our results with different tasks and different populations.

As it is typical in eye tracking studies, our study had a small sample size. The relatively low statistical power of this design may have contributed to non-significant results relating to message source and personal feedback, which have been shown to have significant effects in studies with larger samples [20, 21]. Future studies with larger sample sizes are needed to overcome this limitation.

\section{Conclusion}

The objective of this study was to identify factors that can improve outcomes of health promotion to young adults. We used eye tracking to explore components of an interpersonality model of online persuasion developed for general CMC contexts. The results support the use of eye-tracking in health promotion studies and show that users' eye movement has the potential to serve as a valuable tool in developing experimental treatments and in supporting instrument development and theory building. 


\section{References}

1. Baldwin, M.W., Granzberg, A., Pritchard, E.T.: Cued activation of relational schemas: self-evaluation and gender effects. Canadian Journal of Behavioural Science - Revue Canadienne Des Sciences Du Comportement 35(2), 153-163 (2003)

2. Bendtsen, P., Johansson, K., Åkerlind, I.: Feasibility of an email-based electronic screening and brief intervention (e-SBI) to college students in Sweden. Addictive Behaviors 31(5), 777-787 (2006)

3. Di Meglio, F.: Stress Takes Its Toll on College Students. Bloomberg Businessweek (2012), http://www.businessweek.com/articles/2012-05-10/stresstakes-its-toll-on-college-students

4. Djamasbi, S., Skorinko, J., Siegel, M., Tullis, T.: Online Viewing and Aesthetic Preferences of Generation Y and Baby Boomers: Testing User Website Experience through Eye Tracking. International Journal of Electronic Commerce 15(4), 121-158 (2011)

5. Flora, J.A., Maibach, E.W., Maccoby, N.: The role of media across four levels of health promotion intervention. Annual Review of Public Health 10(1), 181-201 (1989)

6. Gallagher, R.P., Taylor, R.: National survey of counseling center directors (2011); Alexandria, V.A.: International Association of Counseling Service (2010), http: / / www . familyejournal . com/forColleges/1-NSCCD.pdf

7. Gould, S., Arfvidsson, J., Kaehler, A., Sapp, B., Meissner, M., Bradski, G., Baumstarck, P., Chung, S., Ng, A.Y.: Peripheral-foveal vision for real-time object recognition and tracking in video. In: Proceedings of the International Joint Conference on Artificial Intelligence (IJCAI), Hyderabad, India, January 9-12, pp. 23-30 (2007),

https : / / www .aaai .org/Papers / IJCAI / 2007 / IJCAI07-341.pdf

8. Grimes, G.A., Hough, M.G., Signorella, M.L.: Email End Users and Spam: Relationship of Gender and Age Group to Attitudes and Actions. Computers in Human Behavior 23, 318332 (2007)

9. Knopper, M.: Health Promotion? Yeah, There Are Apps for That! Clinician Reviews 20(12), 28-30 (2010)

10. Kwan, M.Y.W., Arbour-Nicitopoulos, K.P., Lowe, D., Taman, S., Faulkner, G.E.: Student reception, sources, and believability of health-related information. Journal of American College Health 58(6), 555-562 (2010)

11. Minkler, M.: Health education, health promotion and the open society: an historical perspective. Health Education \& Behavior 16(1), 17-30 (1989)

12. O'Donnell, M.P.: Definition of Health Promotion 2.0: Embracing Passion, Enhancing Motivation, Recognizing Dynamic Balance, and Creating Opportunities. American Journal of Health Promotion 24(1), iv (2009)

13. Pan, B., Hembrooke, H., Gay, G., Granka, L., Feusner, M., Newman, J.: The determinants of web page viewing behavior: an eye tracking study. In: Spencer, S.N. (ed.) Proceedings of Eye Tracking Research \& Applications, ACM SIGGRAPH, New York (2004)

14. Rayner, K.: Eye movements in reading and information processing: 20 years of research. Psychological Bulletin 124(3), 372-422 (1998)

15. Rayner, K., Smith, T.J., Malcom, G.L., Henderson, J.M.: Eye Movements and Visual Encoding During Scene Perception. Psychological Science 20(1), 6-10 (2009)

16. Stewart-Brown, S., Evans, J., Patterson, J., Petersen, S., Doll, H., Balding, J., Regis, D.: The health of students in institutes of higher education: an important and neglected public health problem? Journal of Public Health 22(4), 492-499 (2000)

17. Storrie, K., Ahern, K., Tuckett, A.: A systematic review: Students with mental health problems-A growing problem. International Journal of Nursing Practice 16(1), 1-6 (2010) 
18. Vertegaal, R., Ding, Y.: Explaining effects of eye gaze on mediated group conversations: amount or synchronization? In: Proceedings of CSCW 2002. ACM Press, New Orleans (2002)

19. WHO, Milestones in health promotion: Statements from Global Conferences, World Health Organization (2009), http://www.who.int/healthpromotion/Milestones_Health_ Promotion_05022010.pdf

20. Wilson, E.V., Djamasbi, S.: Developing and Validating Feedback and Coherence Measures in Computer-Mediated Communication. Communications of the Association for Information Systems (2012a)

21. Wilson, E.V., Djamasbi, S.: Interpersonality and Online Persuasion. In: Proceedings of the 11th HCI in MIS Research Workshop, Orlando, Florid (2012b) 